\title{
Estrategia y proceso estratégico: el camino organizacional hacia el diálogo, la articulación y la innovación
}

\author{
Strategy and strategic process: \\ organizational path towards dialogue, \\ articulation and innovation
}

\section{Claudia Patricia Salas Forero (Colombia)}

pattysf1@yahoo.com

Pontificia Universidad Javeriana

\begin{abstract}
Resumen
El concepto de estrategia es muy común hoy en día en el ámbito no solo de la administración, sino en el de casi todas las disciplinas académicas. La comunicación no es ajena a esta tendencia contemporánea y ha logrado su mayor posicionamiento empresarial u organizacional al ocuparse de estudiar activos intangibles, como marca, reputación, imagen, responsabilidad social, talento o conocimiento.

Alrededor de la estrategia se han creado organizaciones de profesionales,
\end{abstract}

\begin{abstract}
The concept of strategy is very common today not only in the government field, but of almost all academic disciplines. Communication is not immune to this contemporary trend and it has achieved its greatest business or organizational positioning to deal with studying intangible assets such as brand, reputation, image, social responsibility, talent or knowledge. Around the strategy, professional organizations have been created such as the Latin American Forum of Comunicación Strategies, FISEC, in which it was
\end{abstract}

$\begin{array}{lr}\text { FECHA DE RECEPCIÓN: } & 14 \text { de septiembre de } 2012 \\ \text { FECHA DE REVISIÓN: } & 16 \text { de octubre de } 2012 \\ \text { APROBACIÓN: } & 1 \text { de abril de } 2013\end{array}$
Para Citar este artículo / to CIte this ARTicle Salas, C. (2013).Estrategia y proceso estratégico: el camino organizacional hacia el diálogo, la articulación y la innovación. Poliantea (IX), 16, pp. 73-97. 
como la del Foro Iberoamericano de Estrategias de Comunicación, en el que con nueve de sus miembros se realizó el estudio Delphi, expertos provenientes de España y Latinoamérica. Los resultados indican que hasta entre los académicos - profesionales del campo hay confusión sobre lo que es y debe comprender la comunicación estratégica, e indican caminos de estudio futuros, como la necesidad de estudiar la estrategia y sus procesos, pero desde el ser humano relacional o la creatividad e innovación, a pesar del énfasis en la competitividad en otros campos de estudio.

Palabras clave: estrategia, proceso estratégico, comunicación organizacional estratégica, innovación. conducted a Delphi study in nine of its members, experts from Spain and Latin America. The results indicate that even among academics or professionals there is confusion about what strategic communication is and what it should include. It indicates future research paths as the need to study the strategy and processes, but from the human relational or creativity and innovation, despite the emphasis on competitiveness in other fields of study.

Keywords: strategy, strategy process, strategic organizational communication, innovation. 


\title{
Estrategia y proceso estratégico: el camino organizacional hacia el diálogo, la articulación y la innovación
}

\begin{abstract}
Claudia Patricia Salas Forero (Colombia)
Maesta en Enseñanza de la Universidad Marshall (MU), Huntington, Virginia Occidental, Estados Unidos, 2005. Maestra en Ciencias en Relaciones Humanas y Empleados de la Facultad de Negocios, MU, 2004. Maestra en Periodismo con especialización en Relaciones Públicas, MU, 1993 Comunicadora social-periodista, Pontificia Universidad Javeriana, Bogotá, Colombia, 1989. Investigadora de la Universidad Sergio Arboleda y docente de la Pontificia Universidad Javeriana y la Universidad de San Martín, Bogotá, Colombia.
\end{abstract}

\section{Introducción}

El Foro Iberoamericano sobre Estrategias de Comunicación (Fisec) (Tendencias Sociales, 2010) define la estrategia como una ciencia articuladora, para la acción y que busca el diálogo con el fin de escuchar. Esta conceptualización se desea alejar de la visión de la estrategia militar, que marca sus inicios, enfocada en el conflicto.

El objetivo de este estudio es comprender la estrategia, tan común hoy en día, más allá de la administración, hacia los activos organizacionales denominados actualmente intangibles, como lo son la comunicación y su influencia en la reputación o imagen de una organización.

Los nueve expertos del Fisec de este estudio Delphi', como principio del estudio de Delphi, se garantizó anonimato a los intervinientes o expertos provenientes de España, Perú, República Dominicana, Costa Rica, Venezuela y Colombia, miembros y participantes de la versión IX del Fisec, celebrado en Brasil en octubre de 2011, enfocan la estrategia en el ser humano. Como uno de ellos la definiría, "una manera de pensar relacional, no lineal, bajo el principio de la persona, sus procesos socioculturales y su condición de futuro".

1 Como principio del estudio Delphi, se garantizó anonimato a los intervinientes o expertos. 
Como sinónimos de estrategia, tales expertos indican en la segunda fase relacional del cuestionario inicial (realizada por Internet a partir de diciembre de 2011, y personalmente con un solo participante de Colombia), además del futuro o la innovación, el plan, la táctica, la visión, la ruta, la anticipación, la competencia, la semiótica, la reflexión, el sentido, la autopoiesis (sistemas en red que define el acoplamiento de un sistema a su entorno) o estrategar, palabra que usan los miembros del Fisec, para destacar el proceso que sigue la mente humana para hacer una estrategia.

Es decir, en un entorno cambiante, la definición de estrategia está llena de contradicciones, conceptualizada como preservación o transformación, proceso racional o creatividad, cooperación o conflicto, así como presente, pasado y, al mismo tiempo, futuro.

El estudio pretende establecer las bases y el futuro de la estrategia desde la comunicación a través de la mirada de los expertos, porque, a pesar de que los programas académicos en pregrado y posgrado han crecido en esta área en Latinoamérica, sigue habiendo mucha confusión al respecto. Al final, los participantes en el estudio concluyen que la innovación y la creatividad son dos elementos clave en la estrategia, una tendencia que hay que estudiar más a fondo, en cuanto a su relación con la comunicación, así como establecer la cultura organizacional que debe existir para que estas estén presentes.

\section{Metodología}

Estudio Delphi con nueve expertos internacionales provenientes de España y Latinoamérica, miembros y participantes de la versión IX del Fisec, celebrado en Brasil en octubre de 2011.

El estudio tuvo dos fases relacionales: un primera ronda de respuestas presenciales durante el encuentro y una segunda realizada por Internet, a partir de diciembre de 2011, con un solo participante que contestó de forma personal en Colombia.

\section{Marco conceptual}

\section{Estrategia}

El general Baquer, asesor histórico del Instituto Español de Estudios Estratégicos, dice que la estrategia es una teoría frente al conflicto y una forma de pensar. Porque hay que pensar lo que se transmite y lo que se hace (citado por Matilla, 2007). 
Todos los expertos del estudio Delphi coinciden en enfocar la estrategia hacia el futuro, pero, frente a un mundo lleno de cambios, la centran en la comunicación, la educación y el diálogo participativo, una nueva mirada que ha encontrado eco en expertos en el tema, como Pérez (2010), fundador del Fisec.

Sin embargo, ninguno analiza, como señala el gurú Henry Mintzberg, la importancia de los patrones del pasado en las estrategias, ya que el futuro se basa en nuestras experiencias, conocimientos anteriores o hasta en la intuición. Mintzberg indica que estrategia es perpetuación y transformación; diseño con criterio, imaginación intuitiva y aprendizaje emergente; creatividad más allá del proceso racional; comprende el conocimiento individual y la interacción social; la cooperación al igual que el conflicto; tiene que analizar antes, programar después y negociar durante; y todo esto deber ser en respuesta a un entorno que puede ser exigente (Mintzberg, Ashltrand y Lampel, 2003).

Lo anterior está en total acuerdo con las famosas tres cajas del reconocido consultor internacional en innovación y estrategia, Vijay Govindarajan: la caja 1 que es la de administrar el presente o preservar; la caja 2 que es la de olvidar selectivamente el pasado, y la caja 3 que es la de crear futuro o transformarse. Un gerente o presidente de una organización, según Govindarajan y Trimble (2011), debe saber qué destruir del pasado y qué crear para ser un innovador efectivo en estrategia.

En conclusión, en un medio de cambios constantes, la comprensión de la estrategia está llena de oposiciones (ver tabla 1).

Tabla 1. Conceptos de estrategia

\begin{tabular}{|l|l|}
\hline Preservación & Transformación \\
\hline Corto plazo & Largo plazo \\
\hline Imaginación intuitiva & Aprendizaje emergente \\
\hline Proceso racional & Creatividad \\
\hline Crecimiento individual & Interacción social \\
\hline Diseño & Criterio \\
\hline Cooperación & Conflicto \\
\hline Análisis & Negociación y programación \\
\hline Pasado y presente & Futuro \\
\hline
\end{tabular}

Tomado de Mintzberg (2003); Govidarajan y Trimble (2011).

\section{Estrategias y comunicación}

Sobre la relación estrategia-comunicación, los expertos en el estudio Delphi señalan que, dado el carácter relacional de la primera y que esta supone el reconocimiento del otro, la comunicación es básica y es la que favorece la implantación de su plan de acción, indispensable para la participación, conectando el entorno 
necesario para establecer estrategias, ya sea a través de interacciones, medios, mensajes o tecnologías.

Pero la mayoría de los expertos diferencian estrategias de comunicación de comunicación estratégica (7 de 9). Le dan, en general, mayor importancia al término estrategias de comunicación, ya que consideran es la estrategia aplicada a la comunicación; mientras que la comunicación estratégica está dentro de una estrategia, por lo cual varía según la intencionalidad y el contexto. Es decir, el todo es la estrategia de comunicación y la segunda es solo una parte.

Sin embargo, hoy en día, a pesar de que la comunicación es definida más como estratégica y ha adquirido un mayor posicionamiento organizacional o empresarial macro, hay aún confusión respecto de su definición entre expertos iberoamericanos, lo cual se observa hasta con los docentes en las aulas académicas, ya que aún no hay una base teórica clara y compartida por todos.

Uno de los expertos en este estudio, por ejemplo, considera que la primera, la estrategias de comunicación, es más mecánica en el desarrollo del proceso de comunicación, mientras que comunicación estratégica no supone solo medios.

\section{Comunicación estratégica}

Teniendo en cuenta el debate anterior, para definir la comunicación estratégica en conceptos, los expertos en el estudio Delphi usan palabras como un proceso de gestión para crear vínculos o relaciones con públicos de interés, en el cual se alcanzan unos objetivos. Una conceptualización muy similar a la de las relaciones públicas desde la visión global.

También caracterizan la comunicación estratégica como una capacidad, ya sea como parte del ser humano en contexto, o para desarrollar estrategias, la supervivencia y la adaptabilidad: la comunicación estratégica como una parte de la estrategia general, dando respuesta a unos objetivos y opuesta a la información.

La comunicación estratégica, de acuerdo con Pérez (2008), es una forma de acción social que se caracteriza por la interacción simbólica para resolver problemas o maximizar oportunidades, dentro de un juego estratégico, relacionado con un contexto o tendencias, para las que hay que tener en cuenta las posibles decisiones/reacciones de los públicos objetivo, tanto internos como externos. 
Sin embargo, ante la dificultad de aislar la comunicación estratégica como variable de estudio, es considerada, muchas veces, solo como herramienta según un estudio en Chile (Echenique, 2009), con 25 directores de comunicación, los cuales indican que su definición tiene mayor valoración en los activos intangibles, como marca, reputación, imagen, responsabilidad social, talento, conocimiento y capital organizacional, al ser la responsable de generar confianza en las audiencias, pero no posee el peso, por ejemplo, de la reputación, que es un activo contable y transable en el mercado.

Tal afirmación hay que estudiarla más a fondo, para saber si en parte esta es una de las razones por las que en áreas tan importantes para una organización, como la responsabilidad social, no se encuentran comunicadores a cargo, sino administradores o ingenieros, a pesar de ser los primeros fundamentales en el desarrollo e implementación de la comunicación estratégica.

Las estrategias de comunicación son caminos y la comunicación estratégica es redundante

Los expertos
El argumento anterior es similar a las discusiones académicas sobre administración, que consideran que esta de por sí debe ser estratégica.

Un experto piensa, en la segunda ronda del estudio Delphi, que, aunque debería sobrar agregar comunicación a la parte estratégica, se necesita porque a menudo se comunica tan solo en lo táctico, sin formulación previa de objetivos.

Es decir, los expertos (excepto tres), están de acuerdo, en la segunda ronda, en que no toda comunicación es estratégica, ya que, por ejemplo, esta última responde a un diagnóstico, un plan de acción e implementación, como el sentido de la interconexión de la comunicación, y con una evaluación. Uno, por ejemplo, de los que creen que sobra agregar estrategia, considera que lo positivo es la reunión de diversas disciplinas o áreas del conocimiento, como la empresarial, política, psicología o márquetin.

Otro de los expertos incluye la estructura del sentido como parte fundamental para ser estratégicos en la comunicación, dentro de un universo simbólico y de relación doble vía, en la cual no nos enfoquemos únicamente en la unilateralidad del discurso desde la fuente productora. 
Sin embargo, en el futuro, uno de los expertos cree que se llegará a determinar comunicación y estrategia como sinónimos, sobrando indicar esta última para diferenciarla de la parte solo operacional o instrumental. Además, como indica otro de ellos, en general, todas las acciones humanas pueden llegar a considerarse estratégicas porque son relacionales y cognitivas.

\section{Marco teórico}

En un reciente estudio hecho por Torres (2010) sobre la enseñanza universitaria de la comunicación estratégica en España, encontró que las materias de estrategias de comunicación solo se imparten en los estudios de publicidad y relaciones públicas, y que son de carácter profesionalizante y no de disciplina académica, que según la autora, sí es el objetivo de la nueva teoría estratégica.

La estrategia en la comunicación ha adquirido un mayor posicionamiento empresarial $\mathrm{u}$ organizacional, gracias a su valoración actual en activos intangibles, como marca, reputación, imagen, responsabilidad social, talento, conocimiento y capital organizacional (Echenique, 2009).
La nueva teoría estratégica, según Torres (2010), preferencia también los factores relacionales e intangibles sobre los económicos, al permitir que el diseño de estrategias de comunicación sea más acorde con una visión social (humana), poniendo en primer plano los procesos cognitivos o valorativos, es decir, los relacionados con el conocimiento, la moral, la categorización, con el fin de aplicar el saber de forma crítica, o lo que denomina la nueva teoría estratégica como la habilidad de "estrategar", o de liderazgo en la toma de decisiones.

El vacío encontrado por Torres (2010) en cuanto a disciplina académica de la estrategia puede verse reflejado, en parte, en los expertos del estudio Delphi, que no indicaron en la primera ronda de preguntas bases académicas claras, ya que en sus respuestas no hay un conocimiento preciso de la teoría estratégica. Uno solamente, autor del libro $\mathrm{Ha}$ cia una teoría general de la estrategia (Pérez y Massoni, 2009), nombra los siete cambios de la teoría estratégica: la complejidad, el ser relacional, innovación y significación, articulación, comunicación, herramientas como la radiografía analítica del patrón de conectividad, RAPC, que 
es un mapa de interconexiones, flujos, subsistemas y públicos de una organización, o el análisis por dimensiones ya sean informativas, ideológicas, interaccionales o socioculturales, entre otras, y por último los nuevos modelos, como el antropológico, donde hay una unidad central de significación, evaluación e imaginación: el de red de influenciadores y los modelos de comunicación estratégica u organizativa.

Respondiendo, entonces, a un llamado que en 1994 hicieron Prahald y Hammel para que investigadores y estudiosos de la materia repensaran la teoría estratégica desde otros paradigmas, Rafael Pérez en 2001, con su libro Estrategias de comunicación, abrió una puerta del conocimiento al respecto y encontró una respuesta positiva en Iberoamérica (Villegas, 2008), con la propuesta de la nueva teoría estratégica.

Solamente en la segunda ronda del cuestionario, los nueve expertos del estudio Delphi refuerzan los siete cambios de la nueva teoría estratégica como la base teórica para sustentarse, ya que en la primera parte no había mucha claridad entre ellos ni unidad. Otros aspectos que señalan importantes para la estrategia en comunicación son la relación contextual, en la que incluyen la capacidad de adaptarse, así como los públicos, el otro o el entorno, la factibilidad y la aplicación práctica de ella.

\section{Macroestrategia, comunicación y comportamiento}

En un estudio de gerentes o presidentes (CEO's) identificados como excelentes por su retorno en inversión, se encontró que desean una comunicación que sea estratégica, basada en la investigación y que involucre la comunicación de doble vía con sus públicos objetivo (Gerhard y Steyn, 2006). Asimismo, como lo indican estos dos autores, al referirse a la conocida investigación de 103 gerentes generales en Suráfrica, los líderes de las organizaciones esperan que los encargados de la comunicación sean proactivos y desempeñen un papel estratégico en lo macro, interpretando, por ejemplo, las tendencias estratégicas actuales en el medio y guiar sobre esta con relación a la comunicación con los stakeholders (todos los públicos que son o pueden ser afectados por una organización).

En cuanto a las estrategias de comunicación, los expertos miembros o participantes del Fisec en el estudio Delphi nombran aquellas en 
que modifican la actitud del público, las percepciones y las que se centran en el ser humano. Un paso más allá en cuanto a influencia o cambios serían los de comportamiento, de los cuales se les pregunta en la segunda parte de la investigación.

Ante lo anterior, dos de los expertos indican que la influencia en los comportamientos es clara para la comunicación al ser parte fundamental en el proceso de pensamiento/emociones, ya que psicológicamente hablando los seres humanos primero generan percepciones, y es aquí donde es importante abrirse a opciones, luego se generan actitudes acordes con las percepciones y, finalmente, las actitudes inducen a la acción.

Otras estrategias efectivas en comunicación, para cambiar comportamientos, según los expertos, son las del reconocimiento del otro, identificación, consenso y diseminación de los mensajes a los públicos adecuados, que logren un cambio de conciencia en el receptor, o articular los valores compartidos, crear sentido vital para los receptores y considerar la cultura del mensaje. Al indicar ejemplos, uno de ellos señala, los estudios de acción-participación con comunidades, que involucran todos los aspectos anteriores.
Las estrategias anteriores son, en parte, basadas en los principios de persuasión, los cuales señalan que la gente que participa activamente en la toma de decisiones suele conservar cambios de actitud a largo plazo; por lo tanto, al comprometerse no varían de opinión, y son influenciados más rápidamente por líderes en los que confían y creen, dando más credibilidad a la fuente si son repetidos sus mensajes por gente de su comunidad (Xifra y Lalueza, 2009).

\section{El proceso de estrategias de comunicación}

Por otra parte, el proceso que señalan los expertos de una estrategia sí es similar al de la comunicación estratégica, eliminándose el debate anterior sobre si es estrategias de comunicación, o al revés.

Agregan al proceso de estrategia áreas específicas de la comunicación, como el establecimiento y estudio público objetivo, la formulación de un concepto, el diseño de los mensajes o la elección de medios de difusión, incluso las siguientes partes:

1. Una investigación previa (análisis de la situación, antecedentes, documentación, matriz de 
percepciones, benchmarking, búsqueda de opciones, diagnóstico, estudio de públicos).

2. Objetivos.

3. Planificación (formulación de un concepto en comunicación, diseño de los mensajes como patrón de conectividad, análisis de recursos, elección de los medios de comunicación o eje de simetría entre la organización y los públicos, al escucharlos y cambiar según los stakeholders - esta última parte agregada en la segunda ronda por uno de los expertos-, entre otros aspectos).

4. Implementación o la acción (a la cual un experto le agregó en la segunda ronda la ejecución del calendario de actividades y el presupuesto de costos).

5. Evaluación. Esta última parte, al igual que el proceso clásico circular de research, objectives, planning, evaluation, propuesto por Marston (1963), se sugiere que vuelva a los objetivos para comprobar si se cumplieron.
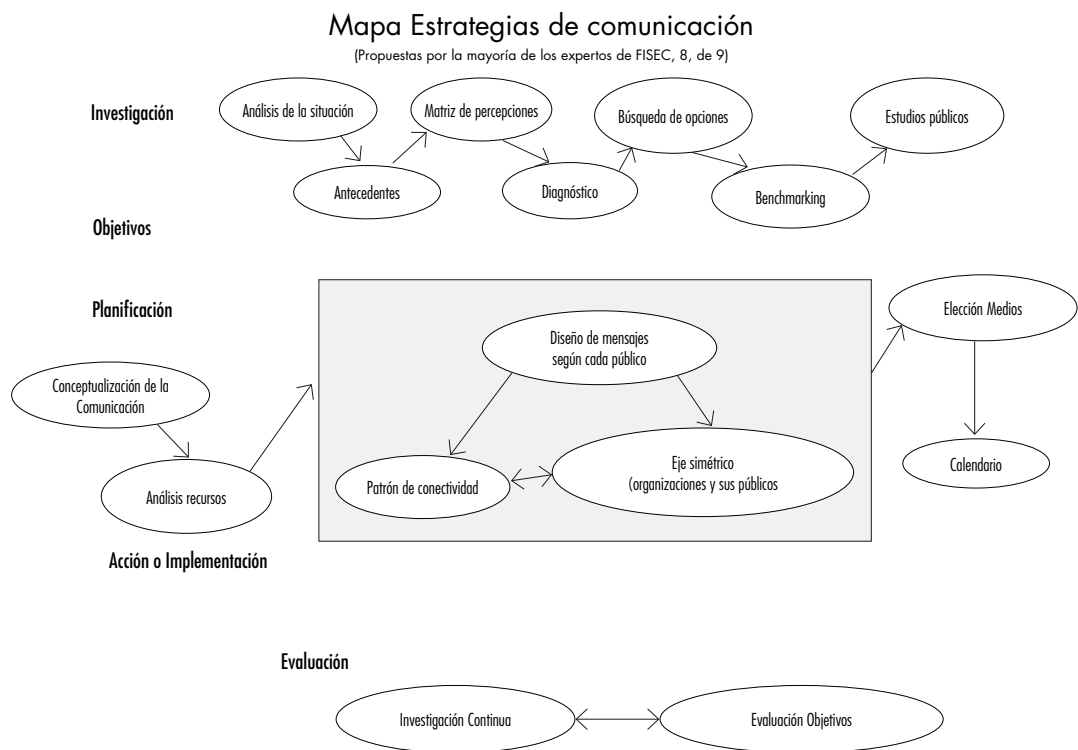

Figura 1. Mapa estrategias de comunicación. 
En esta figura, a pesar de que se plantea un proceso similar al básico de estudio de procesos, es mucho más amplio e incluye aspectos, como la matriz de percepciones, benchmarking o segmentación de públicos, tan acorde con la estrategia actual de competitividad y segmentación de mercados, que va más allá del enfoque en el ser humano relacional de la nueva teoría estratégica de Pérez y Massoni (2009), y que es más práctica con lo que realmente pasa en las organizaciones y sus intereses.
En la planificación, se vuelve a enfocar en la conectividad con segmentación, tanto de medios como mensajes, pero desde un eje simétrico organizacional, que siempre escucha y cambia según sus públicos.

Es más, uno de los expertos, de acuerdo con el anterior proceso de estrategias de comunicación, aportó el siguiente diseño de cuestionamientos como otro apoyo ideal en el manejo de la comunicación estratégica y similar, según a su opinión, al anterior:

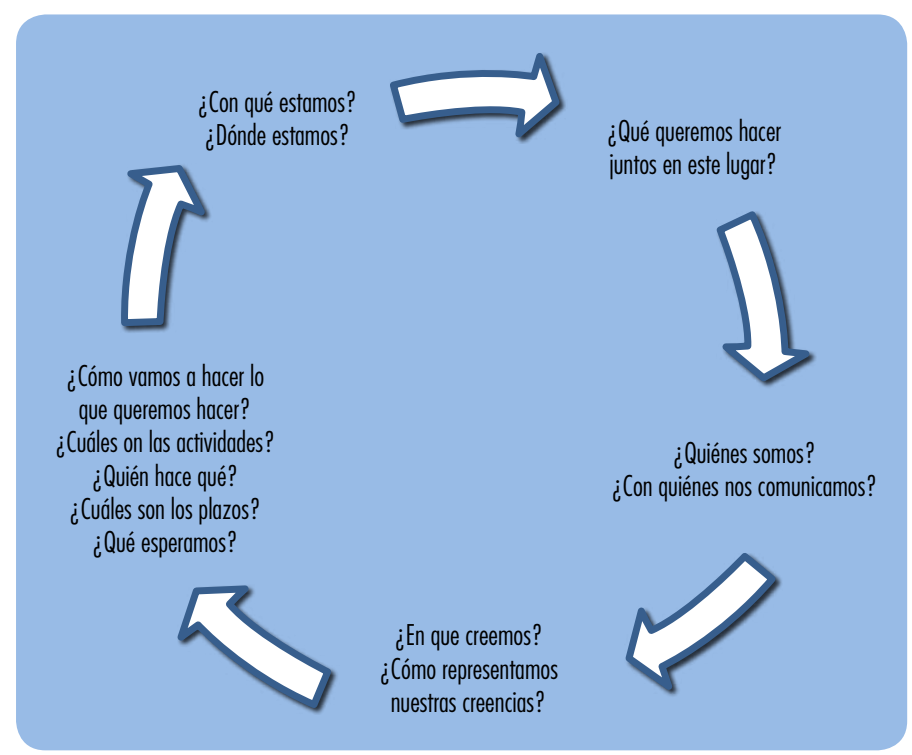

Figura 2. Preguntas al proceso de estrategias de comunicación. Fuente: Realizado por uno de los expertos participante en el estudio Delphi. 
El experto, en cuanto al proceso, indica el siguiente orden en la búsqueda de la resolución de las preguntas anteriores: análisis situacional $\rightarrow$ planteamiento de objetivos $\rightarrow$ determinación del público objetivo $\rightarrow$ construcción del mensaje $\rightarrow$ definición de acciones estratégicas $\rightarrow$ regreso a análisis situacional y seguimiento.

Sin embargo, solo uno de los expertos en la segunda ronda, al indicar el proceso conjunto al que la mayoría llegó, señala no estar de acuerdo con este por ser muy racional y explicar en pocas palabras un modelo que debe ser de operaciones y espiral algorítmico, nombrando las críticas del gurú Henry Mintzberg a las diez principales escuelas de pensamiento estratégico, del cual, en cierta forma, es parte el research, objectives, planning, evaluation.

\section{El proceso estratégico es relacional}

La estrategia de la vida sería, entonces, la que proponen Pérez y Massoni (2009), la ciencia de la relación y la articulación social, poniéndose en el lugar del otro, para ser más dialogantes y consensuales, tratando en la incertidumbre del proceso de llegar a orientaciones correctas, al no haber siempre soluciones exactas.

En cuanto a la relación estrategia y ser relacional, el vínculo es claro para los expertos del estudio Delphi, ya que es el sustento de la nueva teoría estratégica; así no la indiquen específicamente como teoría, sí señalan el hecho de que el ser humano relacional es el centro de todo, con una estrategia que busca relaciones como la razón de su sentido, que permite viabilidad en la práctica y en la cual las elecciones, por ejemplo, dependen de la posición de la organización o de los otros jugadores.

En conclusión, como afirma otro experto, un futuro del estudio del management, pero desde la perspectiva de la nueva teoría estratégica. Se debe, entonces, ser consciente sobre dónde se está y hacia dónde van todas las fuerzas del entorno; ser un hombre relacional pero también aspiracional (sujeto con información, opciones, basado en investigación, evaluación y creatividad), como indicaría Villegas (2008). 


\section{Estrategia y liderazgo}

Un líder sí debe ser estratega, aunque un estratega no necesariamente es líder

Autores como Rodríguez (2008) se refieren a Joan Costa y su propuesta de liderazgo estratégico como uno de los ámbitos profesionales básicos para un comunicador en las organizaciones. Aquel que define la política general de la comunicación como su estrategia favore la construcción de un esquema de imagen, reputación, cambio de cultura, ambiente comprometido y productivo, así como el reconocimiento a largo plazo, al plantear todas las acciones, procesos o relaciones con los diferentes públicos y segmentos de interés.

Sin olvidar la importancia del contexto y la política general de la organización dentro de una función macro del comunicador, todos los expertos del estudio Delphi ven una clara relación entre estrategia y liderazgo; sin embargo, tres de ellos indican que no necesariamente un buen estratega es un líder, porque así logre alcanzar sus metas, el liderazgo debe ser reconocido por los demás, es una creación conjunta de los miembros de una organización, además porque al estratega puede no interesarle ser líder.

En la segunda ronda de preguntas a expertos, la mayoría (ocho de nueve) continúa afirmando que liderazgo y estratega no necesariamente están ligados, ya que este último podría llegar a trabajar aislado de los demás, simplemente planteando opciones estratégicas impulsadas/ejecutadas por otros, o manejar el poder de la influencia y no el directo. Su posible capacidad de liderazgo, en ese caso, no estaría en juego y su capacidad estratégica no se vería afectada.

Otro indica, como ejemplo, el hecho de que hay personas que desarrollan importantes labores de estrategas, pero prefieren mantenerse en el anonimato, o en otros casos, hay estrategas que nunca se conectan como líderes.

Ante lo anterior, otro experto indica que como consultor estratégico una persona puede no ser un líder, pero asesorar a un político, que a su vez es un líder que toma la decisión final, pero no es necesariamente estratega o diseñador de un plan.

Sin embargo, para uno de ellos, aunque a un estratega puede no interesarle ser líder, se tiene que ser para convertirse en estratega, para 
tener credibilidad, criterio y asertividad con la empresa, el cliente o la organización. Ya sea que el liderazgo sea por conocimiento o experiencia, es necesario para veracidad.

En cuanto a otros tres expertos, el estratega o la implementación de una estrategia requiere de por sí liderazgo, para convencer, integrar potencionalidades, buscar opciones y desarrollar competencias tácticas o estratégicas.

La pregunta, o dónde está el cuestionamiento, es que si un estratega no es líder, necesariamente se pueden perder muchos de sus aportes, ya que al ejecutarse por otros o no hacerse su proceso estratégico, como puede suceder, se perdería el gran aporte de su pensamiento en comunicación u otras áreas.

\section{Cambio}

Un dominio de los factores que influyen en los caminos que toma el cambio es el centro de la razón de un sistema estratégico ideal en los seres humanos. De ahí que la planificación y la previsión son otros de los sinónimos que agregaron los expertos a la palabra estrategia en la segunda ronda de preguntas, adicional a la de futuro que ya habían indicado.
Lo cual, en parte, son los desafíos que plantea Manucci (2010), cuando indica que el primero es prepararse para lo desconocido, contextualmente y con posicionamiento; mientras el último o quinto es entrenarse para los tiempos de cambio en el nuevo juego de competitividad, transformando antes lo cotidiano.

Pero para transformarse continuamente, hay que ser conscientes de dónde se está y hacia dónde van todas las fuerzas del entorno, es decir, el hombre relacional y aspiracional.

En una declaración reciente del Fisec (Tendencias21.net, 2010b), académicos de 14 universidades afirmaron:

Los cambios jamás han sido tan rápidos e inesperados y la respuesta que finalmente vayan a dar los operadores estratégicos a esos cambios y a los grandes retos del siglo XXI (salud, educación, desarrollo, migraciones, problemas de género, infancia, relaciones internacionales, paz, generación y reparto de riqueza, etc.), va a depender de la forma en que dichas personas piensen y elaboren sus estrategias. Es decir, que todos vamos a sufrir los errores o a disfrutar los aciertos que resulten de una $u$ otra orientación (p. 1). 
Asimismo, los participantes en indiscutiblemente en el siglo XXI el estudio indican que la estrategia está relacionado con el cambio. son los caminos del futuro, uno que

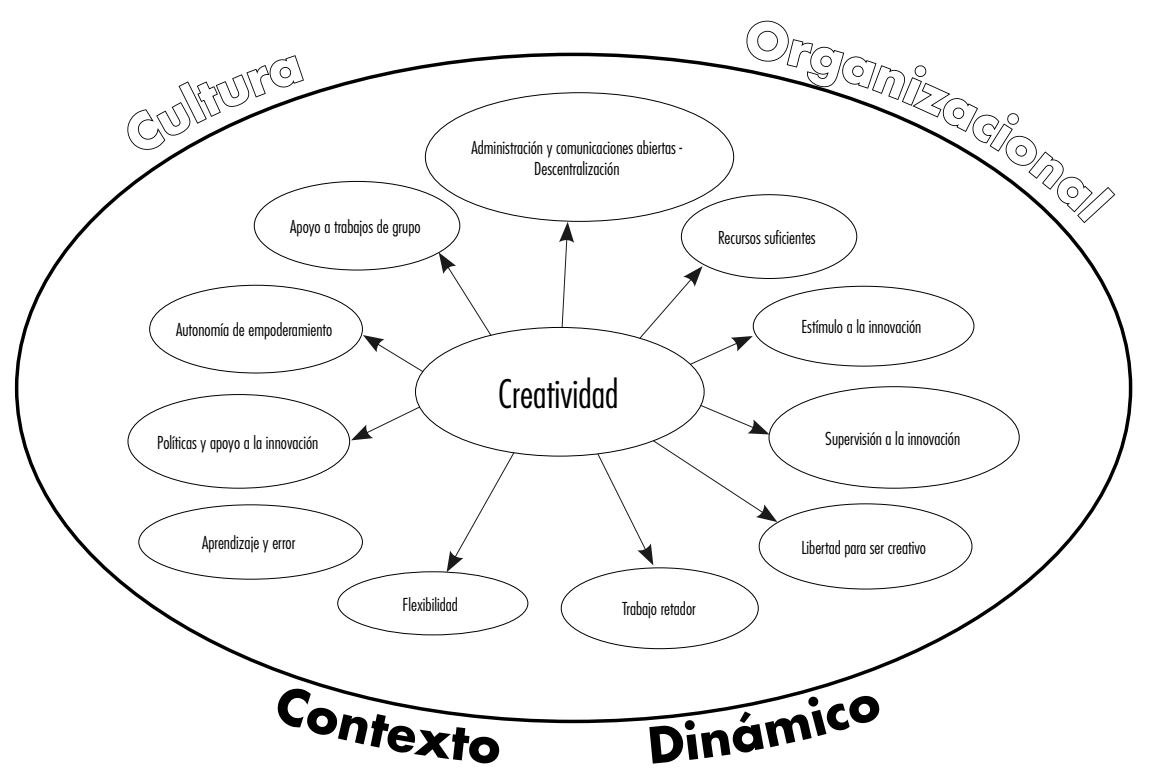

Figura 3. Clima organizacional creativo. Adaptado de Göktan (2005, pp. 1-1 1).

\section{Innovación}

Una estrategia triunfadora es innovadora, porque va más allá de la adaptación o persuasión

Se da por validado así el tercer cambio que propone la nueva teoría estratégica, el cambio en la organización: de unidad de producción a red de innovación y significación (Tendencias21.net, 2010a).

Los tres grandes retos para las compañías en la próxima generación, según el gurú de estrategia en negocios Gary Hamel, serán: innovación, como movilizar la imaginación de cada persona en su organización; adaptabilidad, como construir cosas que se transforman ellas mismas, y compromiso, crear organizaciones que involucran tanto emocional e 
intelectualmente que la gente desee invertir toda su capacidad en el trabajo (Weil, 2007).

Por ejemplo, desde hace una década, la innovación ya se colocaba en los modelos de negocios comerciales por Internet o e-commerce como uno de los factores clave para el éxito de este campo del siglo XXI. Figiel (2005), citando a Thompson y Strickland (2001), indicaba no solo la innovación en los medios para alcanzar una ganancia comprendiendo al público objetivo, sino también la capacidad de adaptabilidad, enfocarse en unas pocas competencias, emplear la última tecnología y asegurar un valor en la cadena de negocios que baje costos o provea un mejor servicio o producto.

Un artículo de Parnell (2010) sobre estrategia en los negocios presenta evidencia de que de los cuatro tipos estratégicos de Miles y Snow señalados en 1978, el de prospectos, es el que ofrece mejor desempeño laboral u organizacional, ya que se es un diseñador y se percibe el medio como dinámico e incierto, manteniendo flexibilidad e innovación; sobre los denominados defensores (buscan control y estabilidad), analizadores (entre estabilidad y flexibilidad) o reactores (sin consistencia en selección estratégica y reaccionan al medio).

Entonces, como respuesta a la pregunta de cierre, la mayoría de los expertos (siete de los nueve) señalan que estrategia e imaginación son sinónimos, porque un estratega debe ver las cosas de forma diferente e innovadora.

Lo anterior se sustenta con la respuesta dada por un experto a la segunda ronda de preguntas del estudio Delphi, en cuanto a la importancia de la creatividad para hacer mejor las cosas, ya que pensar estratégicamente, afirma, es un ejercicio de innovación, tanto en el planteamiento del objetivo, como en la elección de alternativas de acción, y hasta en los momentos de crisis, como confirma otro.

La creatividad en la segunda ronda del estudio, afirman algunos expertos, es tener una visión de la totalidad, del conjunto, de las partes en relación. Fundamental, afirma otro, porque consigue visualizar o llevar a cabo nuevas respuestas.

Sin embargo, uno de ellos aclara que visualiza la innovación como un factor que surge en la estrategia por necesidad, similar a la respuesta de otro participante que indica la existencia de estrategias para 
alcanzar ciertos objetivos, las cuales no implican innovación.

\section{La creatividad y su contexto}

Sin embargo, para ser innovador se necesita el medio adecuado para desarrollarse. Como lo afirma Göktan (2005), la creatividad en los individuos no se da a menos que las organizaciones provean el correcto medio ambiente o clima, tal como estímulo de la organización y supervisión a la innovación; apoyo de trabajo en grupo; libertad para ser creativo; recursos suficientes; un trabajo retador, citando a Amabile (1996) y a Johannessen (1994), sobre comunicaciones abiertas, apoyo desde las políticas organizacionales, estilo de una administración abierta, autonomía, flexibilidad y aprendizaje.

En la mayoría de las empresas $\mathrm{u}$ organizaciones, $\mathrm{y}$ hasta en las mismas facultades académicas donde se enseñan estrategias de comunicación, un sistema alto de centralización en el manejo de información o de decisiones, así como la falta de autonomía en los trabajos individuales o de grupo, afectan negativamente que se lleve a cabo el proceso de la innovación, al ir por varios niveles en la toma de decisiones o solo a través de lo que se llama el "conducto regular".

Las administraciones burocráticas siguen siendo comunes en las grandes organizaciones actuales o en la academia, a pesar de sus obstáculos para la creatividad o innovación, con sus estructuras verticales que eliminan el empoderamiento.

De acuerdo con Isabel López, coordinadora técnica del Informe Anual 2010 de la publicación La comunicación empresarial y la gestión de los intangibles en España y Latinoamérica, "la no tolerancia al error, la visión a corto plazo y la presión de la competencia, son el caldo de cultivo perfecto para los falsos positivos en innovación" (Villafañe, 2010, p. 36).

Pero en lo que sí parece todo el mundo estar de acuerdo en las tendencias actuales o futuras del siglo XXI, incluso los expertos del estudio Delphi, es en la ventaja de actuar con creatividad, para mantenerse en vanguardia, ofrecer el factor sorpresa y ser proactivos. Sin embargo, uno de ellos afirma que en la estrategia pesan más otros aspectos, como la experiencia o el conocimiento, teniendo más importancia la creatividad, por ejemplo, en el diseño o su aplicación. 


\section{Futuro, estrategias \\ y comunicación}

La estrategia implica imaginación creativa para accionary

transformar las propias realidades

Estrategar y futuro son sinónimos. Los expertos definen la capacidad estratégica como la gestión del futuro, adaptándola a los objetivos por alcanzar. Estrategia es anticipación, proactividad, control y elección.

Echavarría (citado por Matilla, 2007) afirma que cuando se habla de estrategia se está discutiendo, necesariamente, sobre el futuro y la comunicación entre personas; por lo tanto, la interrelación de ambas constituye la acción humana permanente. Esta es una de las frases iniciales con las que Matilla, uno de los expertos del estudio Delphi y actual presidenta del Fisec, comienza su tesis de doctorado.

Dicha autora también afirma, en cuanto al futuro de las estrategias de comunicación, que estas deben implementarse como señal de un buen nivel de madurez profesional de los comunicadores, que superen la orientación instrumental o táctica (respuesta a segunda ronda, estudio Delphi, 2011).
El futuro de cuestiones vitales en un mundo de cambio dependerá de la importancia que en las estrategias se les asignen a la comunicación, la educación y al diálogo participativo (Pérez, 2010).

Por lo anterior, todos los expertos, en la segunda fase relacional de las preguntas, predicen un futuro promisorio para la estrategia en las comunicaciones, debido a que constituye la vía más idónea para el consenso, conseguir acuerdos, objetivos y establecer alianzas entre públicos diversos, como la de catedráticos o practicantes profesionales, para una mutua retroalimentación, donde las aulas se conviertan en laboratorios. Asimismo, para la profundización en su estudio, los aumentos de consultoría y, como se indicó anteriormente, para la multidisciplinariedad o conformación de think tanks, donde será más común la existencia de grupos de conocedores de las estrategias de comunicación, con mucha influencia más allá de lo local, tal como los miembros del Fisec.

Un mañana que, de acuerdo con dos expertos, será prometedor, en la medida en que las organizaciones, a través de sus directivos, tomen conciencia de la importancia de la comunicación como una poderosa 
herramienta de gestión y con capacidad de transformar su entorno.

Un buen futuro, que incluye a la estrategia en gestión gerencial, debido a la necesidad de adaptarse a los cambios, el aumento de la capacitación, los estudios de casos reales, la creación de escenarios y una amplia incursión en temas de análisis en la administración o en muchos ámbitos de las crisis (económico, financiero, social, no solo comunicacional); en una era del conocimiento en la que hay que gestionar bien la gerencia con buenas estrategias y hacia la transdisciplinariedad donde el ser humano sea la base del sistema, como eje de intercambios.

Lo que está sucediendo ahora fue parte de las predicciones de Grates (1995) hace más de una década, cuando afirmaba que la naturaleza de la comunicación estaba cambiando, las líneas que separan las disciplinas de la comunicación o la de otros tipos de consultoría, como la administración o la gerencia, estaban desapareciendo como resultado de la rápida transformación en la estructura del mercado, donde se deben crear soluciones innovadoras e interdisciplinarias, siendo más que comunicadores, para convertirnos en gente de negocios, psicólogos, sociólogos o economistas, entre otras áreas.

Un futuro, como indica al final de la segunda ronda del estudio Delphi uno de los expertos, en el cual hay un mayor estudio del management desde la perspectiva de la nueva teoría de la estrategia propuesta en escenarios, como el Fisec.

\section{Resultados}

A continuación, se indican algunas conclusiones del estudio Delphi, en las cuales la mayoría de los expertos coinciden:

Tabla 2. Estudio Delphi

\begin{tabular}{|l|l|}
\hline $\begin{array}{l}\text { La estrategia es relacional y } \\
\text { comunicacional }\end{array}$ & Todos los 9 expertos \\
\hline $\begin{array}{l}\text { La nueva teoría estratégica es } \\
\text { la base teórica de la estrategia } \\
\text { (respuesta dada hasta en la } \\
\text { segunda fase relacional del } \\
\text { estudio) }\end{array}$ & Todos los 9 expertos \\
\hline $\begin{array}{l}\text { Toda comunicación no es } \\
\text { estratégica }\end{array}$ & 6 de los 9 expertos \\
\hline $\begin{array}{l}\text { Diferencia estrategias de } \\
\text { comunicación-comunicación } \\
\text { estratégica }\end{array}$ & 7 de los 9 expertos \\
\hline $\begin{array}{l}\text { Diferencia estrategia y liderazgo } \\
\text { (respuesta dada hasta en la } \\
\text { segunda fase relacional del } \\
\text { estudio) }\end{array}$ & 8 de los 9 expertos \\
\hline Estrategia = innovación & 7 de los 9 expertos \\
\hline
\end{tabular}

Resultados de algunas preguntas hechas a los expertos del estudio Delphi.

Como conclusión, se puede indicar que, de acuerdo con los expertos participantes en el estudio Delphi, la innovación y la 
creatividad son dos elementos clave en la estrategia; y este artículo se centra en dicho argumento, estableciendo su relación y la cultura organizacional que debe existir para que permanezcan, además de otros elementos, como la importancia del pasado o el presente, ya que estrategia no es solo futuro.

De acuerdo con Costa (2001), estimular la creatividad es uno de las funciones de un director de comunicación, que implica a los líderes dentro y fuera de la organización, incluso a todos los stakeholders dentro del plan estratégico, en un proceso de cocreación, tendencia actual en los negocios futuros.

La estrategia de hoy en día se refiere a ser diferente, como lo indica Garrido (2006) citando a Michael Porter. Según este catedrático español, unifica e integra las orientaciones de la empresa, gracias, en gran parte, a la comunicación, y mejora la segmentación, planificando los procesos organizacionales a largo plazo.

En cuanto a las estrategias de comunicación o la comunicación estratégica, todavía falta estudiar y aclarar más en artículos o debates académicos y científicos las teorías en las que se basan, porque se ven vacíos que se reflejan no solo en este estudio, sino en el salón de clases y en los posgrados de universidades, por lo menos latinoamericanas, así se especialicen en el tema.

En el pasado, y aun en el presente, desde frases como que todo o todo el mundo comunica, no se ha posicionado la importancia de la comunicación en las organizaciones y menos en la estrategia empresarial. Los profesionales en el área deben enfocar los problemas de negocios desde las comunicaciones.

La estrategia desde la comunicación se debe comprender en lo macro o hasta cultural, aspectos en los que se deben centrar preguntas o futuros estudios sobre el tema, para aprovechar al máximo su potencial, bajo una visión transy multidisciplinar, que relacionalmente y con indicadores de gestión e innovación comprenda la estrategia proactivamente, situando al ser humano como su eje, pero desde una visión de negocios actual en la que la competitividad es una gran preocupación. 


\section{Referencias}

Amabile, T. M. (1996). Creativity in context. Boulder, CO: Westview Press.

Costa, J. (2001). El director de comunicación. Dirección de comunicación empresarial e institucional. Barcelona: Gestión 2000.

Garrido, F. (2006). Comunicación estratégica. España: Ediciones Gestión 2000.

Echenique, R. (2009, diciembre). Crecimiento y desarrollo de la industria de la comunicación estratégica en Chile. Chasqui, 108, 63-68.

Figiel, V. (2005, enero). A strategy typology and application to e-commerce. Journal of Applied Management and Entrepreneurship, 10(1), 139-150 [en línea]. Consultado el 1 de diciembre de 2011 en <http:// search.proquest.com/docview/203 912923/13442DE69646ED890A9/3 8 ? accountid $=48417>$.

Gerhard, B. y Steyn, B. (2006). Theory on strategic communication management is the key to unlocking the boardroom. Journal of Communication Management, 10(1), 106-109 [en línea]. Consultado el 1 de diciembre de 2011 en <http://search.proquest.com/docvi ew/232932993/1344345547422F677 $14 \mathrm{~F} / 6$ ? accountid $=48417>$.

Göktan, A. (2005). The role of strategy in the innovation process: a stage approach (Tesis de doctorado inédita). University of North Texas, Texas [en línea]. Consultado el 1 de diciembre de 2011 en <http://search. proquest.com/docview/305399718/ 13529DF33312110E70A/1?account $\mathrm{id}=48417>$.

Göktan, A. y Miles, G. (2005). The role of strategy in the innovation process: a stage approach [en línea]. Consultado el 1 de diciembre de 2011 en <http://search.proquest.com/docview/305399718/13 442B2C7B467755244/25?account $\mathrm{id}=48417>$.

Govindarajan, V. y Trimble C. (2011, enero). The CEO's role in business model reinvention. The Magazine. Harvard Business Review [en línea]. Consultado el 1 de febrero de 2012 en <http://hbr. org/2011/01/the-ceos-role-in-business-model-reinvention/ar/1>.

Grates, G. (1995, abril). Communication in the second half of the nineties: strategy. Communication World, 12(4), 16-19 [en 
línea]. Consultado el 1 de diciembre de 2011 en <http://search.proquest.com/docview/210237479/13 4431D19022BDA83BF/1?account id $=48417>$.

Manucci, M. (2010). Contingencias. Bogotá: Norma.

Marston, J. (1963). The nature of public relations. Nueva York: McGraw-Hill.

Matilla, C. (2007). Aportaciones para un modelo global de planificación estratégica en relaciones públicas y comunicación integral. Análisis de un caso: el uso de los modelos de planificación estratégica en algunas agencias y consultoras de relaciones públicas y comunicación (Tesis de doctorado inédita). Universitat Ramon Llull [en línea]. Consultado el 15 de diciembre de 2011en <http:// tdx.cat/handle/10803/350/browse? value $=$ Matilla + Serrano $\% 2 \mathrm{C}+$ Catali na\&type $=$ author $>$.

Mintzberg, H.; Ashltrand, B. y Lampel, J. (2003). Safari a la estrategia. Buenos Aires: Ediciones Granica [en línea]. Consultado el 15 de enero de 2012 en <http://books.google. com.co/books?id=TfYIg8P 1 ybwC \&printsec $=$ frontcover\&dq $=$ mintzb erg+safari+al+estrategia\&hl=es\&s
$\mathrm{a}=\mathrm{X} \& \mathrm{ei}=\mathrm{QmtOT} 6 \mathrm{f} 3 \mathrm{~K} 8 \mathrm{n}$ _ggfwhuz FAg\&ved $=0 \mathrm{CC} 8 \mathrm{Q} 6 \mathrm{AEwAA} \# \mathrm{v}=$ on epage\&q=mintzberg $\% 20$ safari $\% 20$ al\%20estrategia\&f $=$ false $>$.

Parnell, J. (2010). Strategic clarity, business strategy and performance. Journal of Strategy and Management, 3(4), 304-324 [en línea]. Consultado el 15 de diciembre de 2012 en <http://search.proquest.com/docvi ew/761438969/13442B2C7B46775 $5244 / 3$ ? accountid $=48417>$.

Pérez, R. (2008). Estrategias de comunicación. Barcelona: Book Print Digital.

Pérez, R. (2010). Fisec. Carta del presidente. Consultado el $10 \mathrm{de} \mathrm{ju-}$ nio de 2011 en $<$ http://www.fisecforo.org/>.

Pérez, R. y Massoni, S. (2009). Hacia una teoría general de la estrategia. Barcelona: Ariel.

Rodríguez, J. (2008, abril-mayo). Comunicación estratégica. Revista Mexicana de Comunicacion, 20(110), 30-31 [en línea]. Consultado el 15 de diciembre de 2011 en <http://web.ebscohost.com/ehost/ pdfviewer/pdfviewer?vid=12\&hid= 9\&sid=fa1c84bd-8950-4593-b56cc6459eb0a654\%40sessionmgr110>. 
Tendencias21.net (2008). Blog del Foro Iberoamericano sobre Estrategias de Comunicación en Tendencias21. Conclusiones del VI Encuentro Iberoamericano sobre Estrategias de Comunicación [en línea]. Consultado el 10 de junio de 2011 en $<$ http://www.tendencias21. net/fisec/CONCLUSIONES-DELVI-ENCUENTRO-IBEROAMERICANO-SOBRE-ESTRATEGIAS-DE-COMUNICACION_a18. html>.

Tendencias21.net (2010a). Blog del Foro Iberoamericano sobre Estrategias de Comunicación en Tendencias21. Conclusiones del VIII Encuentro Iberoamericano sobre Estrategias de Comunicación [en línea]. Consultado el 20 de noviembre en http://www.tendencias21. net/fisec/CONCLUSIONES-DELVIII-ENCUENTRO-IBEROAMERICANO-SOBRE-ESTRATEGIASDE-COMUNICACION_a28.html.

Tendencias21.net (2010b). Declaración de la isla del pensamiento San Simón [en línea]. Consultado el 10 de junio de 2011 en <http://www.tendencias21.net/Nace-en-Espana-la-Isla-del-Pensamiento_a4634.html>.
Torres Romay, E. (2010, enero/junio). Enseñanza universitaria de la comunicación estratégica en España: análisis de la repercusión del proceso de adaptación al espacio europeo de educación superior en la enseñanza de estrategias. Signo y Pensamiento, 29(56), 304-327 [en línea]. Consultado el 1 de diciembre de 2011 en <http://www. scielo.org.co/scielo.php?pid=S0120$48232010000100018 \&$ script $=$ sci_ arttext>.

Villafañe, J. (2010). La comunicación empresarial y la gestión de los intangibles en España y Latinoamérica. Madrid: Pearson Educación.

Villegas, D. (2008). ¿Se está gestando una nueva teoría estratégica desde Iberoamérica? Revisión y comparación del campo anglosajón e iberoamericano. Revista de Estudios de Comunicación, 14 [en línea]. Consultado el 5 de octubre de en $<$ http:// web.ebscohost.com/ehost/pdfviewer/pdfviewer?vid=12\&hid $=10$ $8 \&$ sid $=$ dd 385a68-ba58-4410-be36$56214545945 \mathrm{e} \% 40$ sessionmgr 111>.

Weil, N. (2007, 15 de noviembre). No Innovation for You! Business strategy guru Gary Hamel blames obsolete management practices for 
your failure to establish a culture of innovation. One solution: stop focusing on efficiency. CIO, 21(4), 1 [en línea]. Consultado el 15 de enero de 2012 en <http://search.proquest.com/docview/201948179/13
44367145B24CECD81/2?account $\mathrm{id}=48417>$.

Xifra, J. y Lalueza, F. (2009). Casos de relaciones públicas y comunicación corporativa. Madrid: Pearson Educación. 
- Estrategia y proceso estratégico - Claudia Patricia Salas Forero 